\title{
Extravasamento do meio de contraste iodado em tomografia computadorizada: uma revisão sistemática de fatores de risco, barreiras utilizadas e tratamentos sugeridos
}

\author{
Iodated contrast overflow in computed tomography: a systematic review of risk factors, \\ barriers used and suggested treatments
}

\author{
Karla Cristina Spadafora ${ }^{\circledR}$, , Erik Lima ${ }^{\circledR}$, Felipe Favaro Capeleti ${ }^{\circledR 1}$, Rafael Eidi Goto ${ }^{\circledR 1}$, \\ Homero José de Farias e Melo ${ }^{\circledR 1}$, Leandro Nobeschi ${ }^{\circledR 1}$
}

\section{Resumo}

Introdução: A utilização de meios de contraste, muitas vezes, permite uma análise mais adequada da morfologia e identificação de doenças. O meio de contraste é comumente administrado por uma bomba de infusão, garantindo fluxo contínuo. No entanto, essa administração automatizada pode aumentar a incidência dos extravasamentos, acarretando a vazão de grandes volumes em um curto período de tempo. Objetivo: correlacionar fatores de risco para o extravasamento do meio de contraste a base de iodo com as barreiras utilizadas e medidas adotadas quando instalada a ocorrência. Métodos: revisão sistematizada utilizando as bases de dados Pubmed e Scielo, nas quais formam analisados 16 artigos dos últimos 10 anos. Resultados: embora pequena a ocorrência, entre 0,01 a 0,09\% dos exames contrastados realizados, o extravasamento é um assunto constante nos centros de imagens radiológicas, sendo de maior incidência em exames de tomografia computadorizada. Um adequado acesso venoso juntamente com meio de contraste que favoreça a infusão estão entre os principais fatores para a transcorrência adequada deste tipo de procedimento. Conclusão: elaboração e aplicação de protocolos que assegurem a não ocorrência, assim como os que abranjam a diminuição das complicações como a síndrome compartimental, proporcionará o aumento de satisfação ao serviço. Casos em que a prevenção não se

1. Faculdade de Ciências Médicas da Santa Casa de São Paulo. Cursos de Graduação em Tecnologia em Radiologia e Sistemas Biomédicos. São Paulo-SP-Brasil

Trabalho realizado: Faculdade de Ciências Médicas da Santa Casa de São Paulo. Cursos de Graduação em Tecnologia em Radiologia e Sistemas Biomédicos. São Paulo-SP-Brasil

Endereço para correspondência: Leandro Nobeschi. Faculdade de Ciências Médicas da Santa Casa de São Paulo. Diretoria de Cursos Tecnológicos. Rua Dr. Cesário Mota Jr, 61-12a andar-01221-020-São Paulo - SP-Brasil. E-mail: leandro.nobeschi@fcmsantacasasp.edu.br faz suficiente, uma adequada abordagem que vise diminuir maiores efeitos indesejáveis, diferenciar o serviço oferecido e a credibilidade dos profissionais envolvidos.

Palavras chave: Tomografia computadorizada, Meios de contraste, Diagnóstico por imagem, Extravasamento de materiais terapêuticos e diagnósticos

\footnotetext{
Abstract

Introduction: The use of contrast media often allows a more adequate analysis of the morphology and identification of diseases. Contrast medium is commonly administered by an infusion pump, ensuring continuous flow. However, this automated administration can increase the incidence of overflows, resulting in the flow of large volumes in a short period of time. Objective: a systematic review of the correlation of risk factors for extravasation of iodine- based contrast medium, with the most used barriers, as well as measures adopted when such an occurrence occurs. Methods: analysis and review of correlated data from PUBMED and Scielo platforms and websites. Eleventh articles were selected, of these, after reading the abstracts and adequacy of objectives, 16 articles from the last 10 years were used. Results: although the occurrence is small, between 0.01 and $0.09 \%$ of contrast-enhanced exams performed, extravasation is a constant issue in radiological imaging centers, with higher incidence in computed tomography exams. The study seeks to elucidate the direct correlation between venous access and the conditions of the contrast medium used, which together favor the infusion, especially when specific risk factors are identified early, thus favoring the proper course of this procedure. Conclusion: elaboration and application of protocols that ensure the non-occurrence, as well as those that cover the reduction of complications such as compartment syndrome, will provide increased satisfaction with the service. In cases where prevention is not enough, an adequate approach aimed at reducing further undesirable effects will differentiate the service offered and the credibility of the professionals involved.
} 
Spadafora KC, Lima E, Capeleti FF, Goto RE, Melo HJF, Nobeschi L. Extravasamento do meio de contraste iodado em tomografia computadorizada: uma revisão sistemática de fatores de risco, barreiras utilizadas e tratamentos sugeridos. Arq Med Hosp Fac Cienc Med Santa Casa São Paulo. $2021 ; 66: e 026$.

Keywords: Computed tomography, Contrast media, Diagnostic imaging, Extravasation of diagnostic and therapeutic materials

\section{Introdução}

Com o avanço da tecnologia nas últimas décadas aumentou-se também a utilização dos exames de imagem, possibilitando assim melhores diagnósticos. Segundo dados do CNES (Cadastro Nacional de Estabelecimentos de Saúde) do Ministério da Saúde, foram contabilizados no estado de São Paulo 1.099 tomógrafos no ano XX, dos quais estão em uso $1.081^{(1)}$. O aumento na realização deste tipo de exame aumenta também a utilização dos meios de contraste (MC) ou radiocontrastantes que são comumente utilizados para o auxílio na detecção das alterações, caracterização e/ ou estadiamento de doenças ${ }^{(2)}$.

Os agentes de radioconstrastes são um tipo de contraste médico utilizado para melhorar a visibilidade interna do corpo e estruturas em técnicas de imagem baseadas em raios $\mathrm{X}$, esses agentes são tipicamente: iodo ou compostos de bário ${ }^{(3)}$.

O contraste iodado pode ser classificado, quanto a sua capacidade de dissociação, em iônico ou não iônico. O contraste iodado iônico é aquele que, quando em solução, dissocia-se em partículas com carga negativa e positiva, enquanto os não iônicos não liberam partículas com carga elétrica. A quantidade de partículas em relação ao volume de solução determina a osmolaridade do contraste. Portanto, o contraste iodado iônico tem maior osmolaridade do que o não iônico. Outras propriedades do contraste dizem respeito à sua densidade e viscosidade. Quanto maior a densidade e a viscosidade, maior será a resistência ao fluxo do contraste, o que torna menor a velocidade de injeção e dificulta sua diluição na corrente sanguínea ${ }^{(4)}$.

Para uma maior qualidade do exame e imagem adequada das estruturas analisadas, é comumente utilizado um dispositivo que administra de forma contínua e com maior rapidez o MC, que se denomina injetora ou bomba de infusão, este garante fluxo contínuo, com pressão precisa durante toda administração, o que possibilita a chegada do MC em todas estruturas do organismo. As taxas de injeção mais rápidas se fazem necessárias para permitir maior realce em algumas situações, aumentando efetivamente a chance de detectar a doença. No entanto, essa administração automatizada pode aumentar a incidência dos extravasamentos, pois permite a vazão de grandes volumes em um curto período de tempo ${ }^{(5)}$.

O extravasamento é definido como administração inadvertida de fluido vesicante no entorno de tecidos saudáveis ao invés do vaso pretendido. $\mathrm{O}$ agente vesicante é um composto que tem a capacidade de provocar bolhas ou necrose tecidual ${ }^{(6)}$.

Embora benéfico para a garantia de um diagnóstico de maior eficácia, a ocorrência de extravasamentos do meio de contraste (EMC) em exames de tomografia computadorizada (TC) se faz presente nos centros de radiodiagnóstico ${ }^{(2,6-13)}$. Busca-se correlacionar os fatores de risco existentes para o EMC a base de iodo com as barreiras utilizadas para a prevenção e medidas adotadas quando instalada a ocorrência.

\section{Método}

Para realização da pesquisa foram utilizados dados on-line do Ministério da Saúde artigos da base de dados da Scielo. Como base de dados utilizou-se o site Pubmed, onde foi realizada revisão da literatura com os seguintes descritores: tomografia computadorizada (Tomography, $x$-ray), extravasamento de material em terapia diagnóstica (Extravasation of diagnostic and therapeutic materials), meio de contraste (Contrast media) e diagnóstico por imagem (Diagnostic imaging). Os critérios de inclusão para pesquisa foram: artigos com textos completos, realizados somente em seres humanos e dos últimos 10 anos. Após a realização da pesquisa 214 artigos foram encontrados, destes com a aplicação dos filtros acima citados restaram 110, que após leitura dos resumos e aplicação de critérios de elegibilidade por afinidade com o título, obteve-se 16. A figura 1 ilustra de forma simplificada e organizada esse processo.
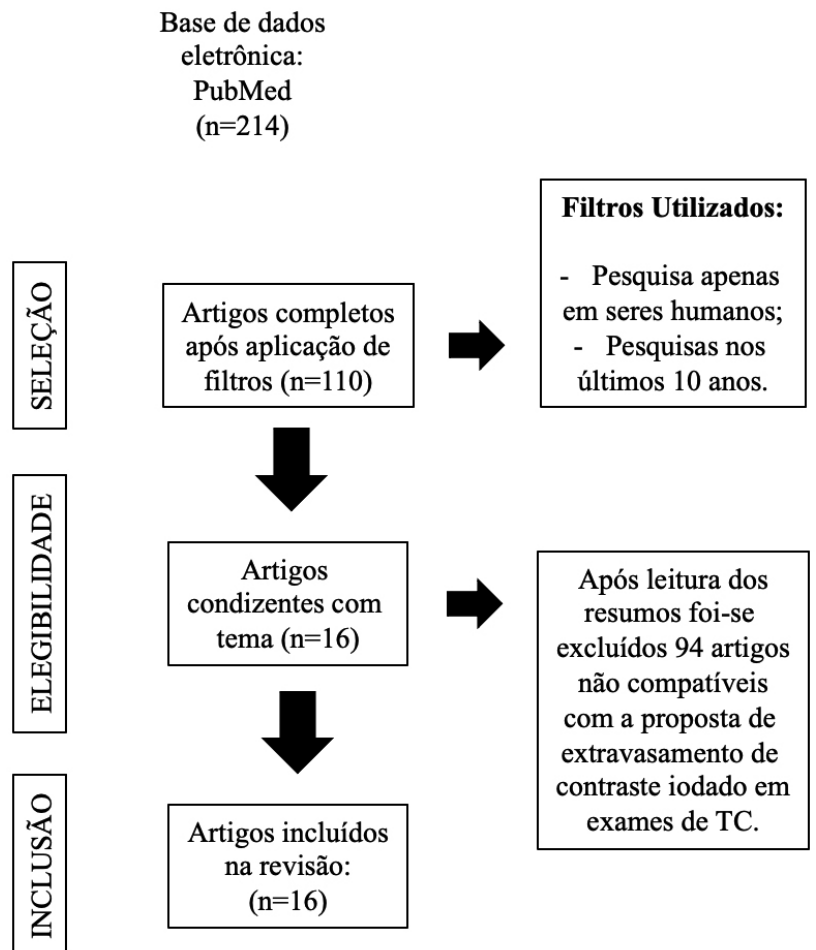

Figura 1 - Organograma com critérios utilizados

\section{Resultados}

O quadro a seguir demonstra a relação de artigos quanto os fatores de risco e conclusões. 


\begin{tabular}{|c|c|c|}
\hline \multicolumn{3}{|c|}{ Quadro 1} \\
\hline Autor/ano/Titulo do artigo & Fatores de risco para EMC & Conclusão \\
\hline $\begin{array}{l}\text { Chad et al, } 2009^{(10)} \\
\text { Experimetal detection of subcutanious } \\
\text { contrast, extravasations using } \\
\text { radiofrequency permittivity sensing. }\end{array}$ & Não se aplica. & $\begin{array}{c}\text { Detecção por radiofrequência é ideal } \\
\text { por não alterar resultado por conta } \\
\text { do aquecimento do contraste. }\end{array}$ \\
\hline $\begin{array}{c}\text { Sbytany et al, } 2010^{(9)} \\
\text { CT contrast extravasation in the } \\
\text { upper extremity: strategies for } \\
\text { managent. }\end{array}$ & Uso de contraste iônico & $\begin{array}{l}\text { Meios de contraste não iônicos } \\
\text { trouxeram melhoras significativas } \\
\text { nas complicações e nos EMC }\end{array}$ \\
\hline $\begin{array}{c}\text { Wienbeck et al, } 2010^{(15)} \\
\text { Prospective study of access } \\
\text { complications of automated contrast } \\
\text { injection with peripheral venous } \\
\text { access in MDCT. }\end{array}$ & $\begin{array}{l}\text { Calibre do dispositivo; } \\
\text { Localização do acesso venoso. }\end{array}$ & $\begin{array}{l}\text { Estudo evidencia a localização do } \\
\text { acesso venoso em extremidades } \\
\text { como fator predisponente para } \\
\text { EMC. Não provou relação com uso } \\
\text { de injetora de infusão ou com a } \\
\text { concentração do iodo. } \\
\end{array}$ \\
\hline $\begin{array}{l}\text { Belzunegui et al, } 2011^{(3)} \\
\text { Extravasation of radiographic contrast } \\
\text { material and compartment syndrome } \\
\text { in the hand: a case report. }\end{array}$ & $\begin{array}{l}\text { Uso de injetora de infusão; } \\
\text { Paciente com acesso venoso frágil; } \\
\text { Paciente com insuficiência venosa }\end{array}$ & $\begin{array}{c}\text { EMC embora raro ocorre } \\
\text { principalmente nos casos de } \\
\text { utilização de bombas injetoras }\end{array}$ \\
\hline $\begin{array}{c}\text { Rowlett, } 2012{ }^{(11)} \\
\text { Extravasation of contrast } \\
\text { media managed with recombinant } \\
\text { human hyaluronidase. }\end{array}$ & $\begin{array}{l}\text { Dados limitados sobre uso de } \\
\text { hialuronidase em EMC. }\end{array}$ & $\begin{array}{l}\text { A hiluronidase é uma opção de } \\
\text { tratamento razoável para extensos } \\
\text { EMC, porém é pouco divulgada. }\end{array}$ \\
\hline $\begin{array}{c}\text { Tonolini et al, } 2012^{(13)} \\
\text { Extravasation of radiographic contrast } \\
\text { media: prevention, diagnosis and } \\
\text { treatment. }\end{array}$ & $\begin{array}{c}\text { Crianças pequenas, idosos não } \\
\text { colaborativos e inconscientes. } \\
\text { Obesos ou com câncer em } \\
\text { quimioterapia. } \\
\text { Uso de bomba injetora de infusão, } \\
\text { particularmente durante aquisições } \\
\text { de múltiplos detectores para estudos } \\
\text { angiográficos. }\end{array}$ & $\begin{array}{c}\text { Recomendável adoção de protocolos } \\
\text { sistemáticos em abordagens } \\
\text { preventivas, diagnósticas e } \\
\text { terapêuticas. }\end{array}$ \\
\hline $\begin{array}{c}\text { Devenport et al, } 2012^{(16)} \\
\text { Rate of contrast material } \\
\text { extravasations and allergic-like } \\
\text { reactions affect of extrinsic warming } \\
\text { of low-osmolality iodinated CT } \\
\text { contrast material to } 37^{\circ} \mathrm{C} \text {. }\end{array}$ & $\begin{array}{l}\text { Estudo sobre a viscosidade o MC } \\
\text { Iopamidol®. }\end{array}$ & $\begin{array}{c}\text { O aquecimento extrínseco }\left(\text { a } 37^{\circ} \mathrm{C}\right) \\
\text { não parece afetar taxas de eventos } \\
\text { adversos para injeções intravenosas } \\
\text { de Iopamidol } 300 \AA \text { a menos de } \\
6 \mathrm{~mL} / \mathrm{s} \text {, mas esta associada a } \\
\text { uma redução significativa no } \\
\text { extravasamento e evento adverso } \\
\text { geral. }\end{array}$ \\
\hline $\begin{array}{l}\text { Kingston et al, } 2012^{(17)} \\
\text { Study of patients with intravenous } \\
\text { contrast extravasation o CT studies, } \\
\text { with radiology staff and ward staff } \\
\text { cannulations. }\end{array}$ & $\begin{array}{l}\text { Sugere possibilidade de diferenças } \\
\text { de ocorrências em manejo de acesso } \\
\text { venoso por equipes distintas. }\end{array}$ & $\begin{array}{l}\text { Pessoal pertencente ao setor de } \\
\text { radiologia traz maior segurança e } \\
\text { prevenção de ocorrências. } \\
\text { Modificação na quantidade de } \\
\text { ocorrências está relacionada também } \\
\text { ao tamanho e calibre da cânula. }\end{array}$ \\
\hline $\begin{array}{l}\text { Yurdakul et al, } 2014^{(2)} \\
\text { Compartment syndrome due to } \\
\text { extravasation of contrast material: a } \\
\text { case report. }\end{array}$ & $\begin{array}{c}\text { Acesso em dorso de mão. } \\
\text { Não notificação de desconforto por } \\
\text { paciente. } \\
\text { Uso de bomba injetora de infusão. } \\
\text { Aumento de uso dos exames de TC } \\
\text { para varredura e estadiamento de } \\
\text { paciente com câncer. }\end{array}$ & $\begin{array}{l}\text { Pode-se reduzir o risco da ocorrência } \\
\text { com uso de contraste do tipo } \\
\text { não- ionico, uso de dispositivos } \\
\text { que detectam extravasamento } \\
\text { precocemente por impedância, } \\
\text { eleição de acesso venoso em vasos } \\
\text { mais calibrosos. }\end{array}$ \\
\hline $\begin{array}{c}\text { Shaqdan, } 2014^{(6)} \\
\text { Incidence of contrast medium } \\
\text { extravasation for CT and MRI a large } \\
\text { academic medical center: a report on } \\
502,391 \text { injections. }\end{array}$ & $\begin{array}{l}\text { Pacientes acima de } 60 \text { anos e } \\
\text { internados. } \\
\text { Uso de bomba injetora de infusão }\end{array}$ & $\begin{array}{c}\text { Pacientes submetidos a TC } \\
\text { apresentam maior risco de EMC dos } \\
\text { que realizam RM. Mais provável } \\
\text { em pacientes acima de } 60 \text { anos e } \\
\text { que recebam o contraste de bomba } \\
\text { injetora de infusão. }\end{array}$ \\
\hline
\end{tabular}




\begin{tabular}{|c|c|c|}
\hline \multicolumn{3}{|c|}{ Quadro 1} \\
\hline Autor/ano/Titulo do artigo & Fatores de risco para EMC & Conclusão \\
\hline $\begin{array}{l}\text { Thomas et al, } 2014^{(14)} \\
\text { Intravenous contrast extravasation } \\
\text { during CT: a national data registry } \\
\text { and practence quality improvemet } \\
\text { initiative. }\end{array}$ & $\begin{array}{l}\text { Acesso venoso difícil ou múltiplas } \\
\text { tentativas. } \\
\text { Pacientes com insuficiência venosa ou } \\
\text { linfática. }\end{array}$ & $\begin{array}{c}\text { Estudo confirma dados anteriores: } \\
\text { taxa de EMC } 0,15-0,70 \% \text {. Volumes } \\
\text { entre } 10-50 \mathrm{ml} \text {, causando lesões leves. } \\
\text { O estudo não concorda com uso de } \\
\text { volumes limite. }\end{array}$ \\
\hline $\begin{array}{c}\text { Alami et al, } 2015^{(12)} \\
\text { Extravasation of contrast medium } \\
\text { during CT } \\
\text { examination: an observational case - } \\
\text { control study. }\end{array}$ & $\begin{array}{l}\text { Volume administrado. } \\
\text { Uso de bomba de infusão } \\
\text { Comorbidades }\end{array}$ & $\begin{array}{l}\text { Uma baixa taxa de injeção é um fator } \\
\text { de proteção contra EMC. O estudo } \\
\text { sugere que pacientes com doenças } \\
\text { cardíacas são mais predispostos a } \\
\text { EMC do que outros. }\end{array}$ \\
\hline $\begin{array}{c}\text { Kim et al, } 2016^{(5)} \\
\text { Computed tomography contrast } \\
\text { media extravasation: treatmetalgorit } \\
\text { m by squeezing with multiple seit } \\
\text { incisions. }\end{array}$ & $\begin{array}{l}\text { Uso de injetora de infusão. } \\
\text { Impossibilidade de acompanhamento } \\
\text { dos pacientes ambulatoriais. }\end{array}$ & $\begin{array}{l}\text { Aplicação de protocolo que assegure } \\
\text { diminuição de complicações de } \\
\text { prevensão de efeitos como síndrome } \\
\text { compartimental. }\end{array}$ \\
\hline $\begin{array}{l}\text { Refky et al, } 2016^{(7)} \\
\text { Contrast media extravasation of } \\
\text { computed tomography and magnetic } \\
\text { resonance imaging: management } \\
\text { guidelines for the radiologist. }\end{array}$ & $\begin{array}{l}\text { Crianças, mulheres e idosos em uso } \\
\text { de quimioterapia. } \\
\text { Pacientes internados. } \\
\text { Acessos venoso com múltiplas } \\
\text { tentativas. }\end{array}$ & $\begin{array}{l}\text { Embora extravasamentos sejam } \\
\text { pouco frequentes os radiologistas } \\
\text { devem estar familiarizados para } \\
\text { evitar complicações severas. }\end{array}$ \\
\hline $\begin{array}{c}\text { Tonoline, } 2016^{(13)} \\
\text { Contrast médium extravasation: } \\
\text { the importance of radiographic } \\
\text { assessment. }\end{array}$ & $\begin{array}{l}\text { Queixas inespecíficas e achados } \\
\text { físicos que não prevê de forma } \\
\text { confiável a verdadeira entidade do } \\
\text { EMC para terapia específica }\end{array}$ & $\begin{array}{l}\text { Importância da diferenciação do } \\
\text { EMC, pratica recomendada na } \\
\text { última versão (versão 9.0) das } \\
\text { Diretrizes Europeias. }\end{array}$ \\
\hline $\begin{array}{c}\text { Behzadi et al, } 2018^{(18)} \\
\text { MRI and CT contrast media } \\
\text { extravasation. A systematic review. }\end{array}$ & $\begin{array}{l}\text { Mulheres, pacientes acima de } 60 \text { anos. } \\
\text { Uso de cateteres } \\
\text { Calibre inadequado do vaso } \\
\text { Punções múltiplas }\end{array}$ & $\begin{array}{c}\text { O EMC é maior em TC, idade } \\
\text { avançada, sexo feminino e acessos } \\
\text { venoso inadequados juntamente ao } \\
\text { uso de injetora de infusão. O não } \\
\text { aquecimento do contraste influencia } \\
\text { diretamente nas ocorrências }\end{array}$ \\
\hline $\begin{array}{c}\text { Wienbeck et al, } 2010^{(15)} \\
\text { Prospective study of access } \\
\text { complications of automated contrast } \\
\text { injection with peripheral venous } \\
\text { access in MDCT. }\end{array}$ & $\begin{array}{l}\text { Calibre do dispositivo e localização } \\
\text { do acesso }\end{array}$ & $\begin{array}{l}\text { Estudo evidencia a localização do } \\
\text { acesso venoso em extremidades } \\
\text { como fator predisponente para } \\
\text { EMC. Não provou relação com uso } \\
\text { de injetora de infusão ou com a } \\
\text { concentração do iodo. }\end{array}$ \\
\hline
\end{tabular}

\section{Discussão}

Segundo estudos recentes os EMC são de maior incidência em exames de TC em comparação a outros métodos de imagem como a ressonância magnética, por exemplo. Os principais fatores de risco como: a idade avançada, sexo feminino, uso de acesso venoso já existente no lugar de uma punção especifica para procedimento, paciente em estado de internação prolongada, uso de injetora de infusão na administração do MC, altas taxas de punção, localização do acesso venoso e o não aquecimento dos MC mais $\operatorname{viscosos}^{(14-15,18)}$.

Em uma pesquisa retrospectiva com 502.391 injeções de MC, confirmou a maior probabilidade de ocorrência com o uso da injetora de infusão, principalmente em pacientes acima de 60 anos. ${ }^{(6)}$ Corroborando a esses achados Belzunegue et al, ${ }^{(3)}$, apresenta que em condições predisponentes como: o uso de injetora em pacientes com idade avançada e ou com acessos venosos em extremidades, sugestivos de fragilidade devem ser monitorados ativamente, assim como uma adequada instrução deve ser dispensada a este paciente visando o relato de qualquer desconforto durante a injeção do MC. A instrução de todos os passos do procedimento juntamente com a caracterização adequada de possíveis sinais causados pelo uso do contraste no organismo, beneficia o paciente tanto na não ocorrência como no possível EMC; tendo em vista que a quantidade do $\mathrm{MC}$ extravasado influenciará diretamente no grau de possíveis lesões.

Por meio dos achados, observa-se que pacientes portadores de doenças cardíacas são mais predispostos ao EMC do que outros, isto está de acordo com o fato de que doenças cardiovasculares aumentam a fragilidade vascular ${ }^{(12)}$.

Entende-se que um adequado acesso venoso juntamente com MC que favoreça a infusão estão entre os principais 
Spadafora KC, Lima E, Capeleti FF, Goto RE, Melo HJF, Nobeschi L. Extravasamento do meio de contraste iodado em tomografia computadorizada: uma revisão sistemática de fatores de risco, barreiras utilizadas e tratamentos sugeridos. Arq Med Hosp Fac Cienc Med Santa Casa São Paulo. $2021 ; 66: e 026$.

fatores para a transcorrência adequada deste tipo de procedimento. $\mathrm{O}$ aquecimento extrínseco dos $\mathrm{MC}$ mais viscosos, realizado geralmente em estufas a $37^{\circ} \mathrm{C}$ diminuem a barreira causada pela alta viscosidade, assim como o uso dos compostos não- iônicos aqueles com menor concentração de moléculas de iodo também diminuem as ocorrências e os eventos adversos causados pelo contato deste com tecidos adjacentes quanto ocorrido o $\operatorname{EMC}^{(2,16)}$.

Embora a ocorrência dos EMC seja pequena, é um assunto constante nos centros de imagens radiológicas, a literatura destaca ocorrências entre 0,01 a $0,09 \%$ dos exames contrastados realizados.

$\mathrm{Na}$ última década houve uma queda significativa tanto de ocorrências como de complicações, pois tornou-se padrão a utilização de MC não-iônicos o que confirma que seu uso também é favorável na prevenção ${ }^{(9)}$. A importância do conhecimento dos fatores de risco, mecanismos de danos teciduais e possíveis consequências para a equipe atuante no setor de imagem $^{(13)}$. Uma abordagem realizada por pessoal devidamente treinado, ou seja, pertencente ao setor de radiologia traz maior segurança e prevenção de ocorrências ${ }^{(17)}$.

Embora a maioria das ocorrências resolva-se com abordagem conservadora, a equipe atuante deve saber quando uma intervenção mais ampla se faz necessária, como a abordagem por especialista vascular, por exemplo ${ }^{(7)}$.

Tonoline $^{(13)}$ ressalta que uma das recomendações das Diretrizes da Sociedade Europeia de Radiologia Urogenital (versão 9,0) é a correta e adequada diferenciação do EMC, que possibilitará uma abordagem personalizada para cada característica apresentada.

\section{Conclusão}

O EMC iodado usando um injetor de infusão é uma complicação comum relatada nas práticas radiológicas, embora sua ocorrência esteja entre $0,01 \%$ e $0,09 \%$ de todos os exames contrastados, deve receber lugar de destaque entre os assuntos pertinentes ao serviço. Os extravasamentos são considerados eventos adversos e devem ser monitorados ativamente, pois são uma importante ferramenta de medição de qualidade por estarem ligados ativamente ao índice de satisfação do usuário, que indicará sua fidelização ou não ao serviço.

Protocolos que orientem quanto a padronização de procedimentos e que visem evitar os extravasamentos são despendidos nestes serviços todos os dias e a cada exame. Porém, na ótica dos usuários, a visibilidade do assunto só se dá nos casos de extravasamentos já instalados, ou seja, durante a abordagem na ocorrência. Por isso, tão importante quanto prevenir a ocorrência é também prestar uma adequada assistência durante os EMC.

Diversas manobras são estudadas e utilizadas no momento, destaca-se neste estudo o uso da enzima Hialuronidase como opção de tratamento para o EMC extenso, essa técnica pouco divulgada na literatura melhora os sintomas e garante o retorno, após administração via subcutânea, dos efeitos causados pós EMC.

A elaboração e aplicação de protocolos que assegurem a não ocorrência, assim como os que abranjam a diminuição das complicações como a síndrome compartimental, gerará o aumento de satisfação ao serviço. Casos em que a prevenção não se faz suficiente, uma adequada abordagem que vise diminuir maiores efeitos indesejáveis, diferenciará o serviço oferecido e a credibilidade dos profissionais envolvidos.

Entende-se que tais protocolos devem conter prazos de atualização com a renovação de dados pertinentes ao assunto. Os profissionais envolvidos no serviço de diagnóstico por imagem, enfermeiros, técnicos e tecnólogos em radiologia deve receber suporte de conhecimento pertinente à identificação, prevenção e atuação nos EMC periódicos. Sugere-se simulações periódicas sobre o assunto, que visem o esclarecimento e direcionamento adequado de acordo com as diversas possibilidades, e que abranjam os diversos profissionais ligados intimamente a este procedimento.

\section{Referências}

1. Brasil. Ministério da Saúde. DATASUS. CNES - Cadastro Nacional de Estabelecimentos de Saúde - Recursos Físicos. [Internet]. [citado 2020 Abr 22]. Disponível em: https:/ / datasus. saude.gov.br/cnes-recursos-fisicos/

2. Yurdakul E, Salt Ö, Durukan P, Duygulu F. Compartment syndrome due to extravasation of contrast material: a case report. Am J Emerg Med. 2014; 32(9):1155.e3-5.

3. Belzunegui T, Louis CJ, Torrededia L, Oteiza J. Extravasation of radiographic contrast material and compartment syndrome in the hand: a case report. Scand J Trauma Resusc Emerg Med. 2011; $19: 9$

4. Juchem BC, Dall'Algnol CM, Magalhães AMM. Contraste iodado em tomografia computadorizada: prevenção de reações adversas. Rev Bras Enferm. 2004; 57(1):57-61.

5. Kim SM, Cook KH, Lee IJ, Park DH, Chul Park M. Computed tomography contrast media extravasation: treatment algorithm and immediate treatment by squeezing with multiple slit incisions. Int Wound J. 2016; 14(2):430-4.

6. Shaqdan K, Aran S, Thrall J, Abujudeh H. Incidence of contrast medium extravasation for CT and MRI in a large academic medical center: a report on 502,391 injections. Clin Radiol 69. 2014; 69(12):1264-72.

7. Refky NMS, Khalid WS, Shima A, Aran S, Prabhakar AM, Singh AK, et al. Contrast media extravasation of computed tomography and magnetic resonance imaging: management guidelines for the radiologist. Curr Probl Diagn Radiol. 2016; 45(3):161-4

8. Massimo T. Contrast medium extravasation: the importance of radiographic assessment. Curr Probl Diagn Radiol. 2016; 45(3):236-7.

9. Sbitany H, Koltz PF, Mays C, Girotto JA, Langstein HN. CT contrast extravasation in the upper extremity: strategies for management. Int J Surg. 2010; 8(5):384-6.

10. Chad E, Bouton MS, Lombardi T, Hobson FR, Stark G. Experimental detection of subcutaneous contrast extravasation using radio frequency permittivity sensing. J Comput Assist Tomogr. 2009; 33(6):824-7. 
11. Rowlett J. Extravasation of contrast media managed with recombinant human hyaluronidase. Am J Emerg Med. 2012; 30(9):2102.e1-3.

12. Alami Z, Nasri S, Ahid S, Kacem HH. Extravasation of contrast medium during CT examination: an observational case-control study. Pan Afr Med J. 2015 30; 20:89.

13. Tonollini M, Campari A, Bianco R. Extravasation of radiographic contrast media: prevention, diagnosis, and treatment. Curr Probl Diagn Radiol. 2012; 41(2):52-5.

14. Dykes TM, Bhargavan-Chatfield M, Dyer RB. Intravenous contrast extravasation during a national data registry and practice quality improvement initiative. J Am Coll Radiol. 2015; 1(2):183-91.

15. Wienbeck S, Fischbach R, Kloska SP, Seidensticker P, Osada N, Heindel W, et al. Prospective study of access site complications of automated contrast injection with peripheral venous access in MDCT. AJR Am J Roentgenol. 2010; 195(4):825-9.
16. Davenport MS, Wang CL, Bashir MR, Neville AM, Paulson EK. Rate of contrast material extravasations and allergiclike reactions: effect of extrinsic warming of low-osmolality iodinated CT contrast material to $37^{\circ} \mathrm{C}$. Radiology. 2012; 262(2):475-84

17. Kingston RJ, Young N, Sindhusake DP, Truong M. Study of patients with intravenous contrast extravasation on CT studies, with radiology staff and ward staff cannulations. J Med Imaging Radiat Oncol. 2012; 56(2):163-7.

18. Behzadi AH, Farooq Z, Newhouse JH, Prince MR. MRI and CT contrast media extravasation a systematic review. Medicine. 2018; 97(9):e0055.

Trabalho recebido: $08 / 11 / 2020$

Trabalho aprovado: 23/08/2021

Trabalho publicado: 09/09/2021

Editor Responsável: Prof. Dr. Eitan Naaman Berezin - Editor Chefe 